\title{
Hydrologie et hydrochimie des eaux dans la zone de construction du chenal du port de pêche de Grand-Lahou, Côte d'Ivoire
}

\author{
Kouakou Séraphin KONAN ${ }^{1,3 *}$, Kouakou Lazare KOUASSI ${ }^{1}$, \\ Kouassi Innocent KOUAME ${ }^{2}$, Aka Marcel KOUASSI ${ }^{3}$ et Dago GNAKRI ${ }^{1}$ \\ ${ }^{1}$ Université Jean Lorougon Guédé, BP 150 Daloa, Côte d'Ivoire. \\ ${ }^{2}$ Université Nangui Abrogoua, 01 BP 10588 Abidjan 01, Côte d'Ivoire. \\ ${ }^{3}$ Centre de Recherches Océanologiques, BP V18 Abidjan, Côte d'Ivoire. \\ *Auteur correspondant,E-mail : konandks@yahoo.fr
}

\section{RESUME}

Une caractérisation des eaux de l'estuaire de Grand-Lahou a été entreprise pour accompagner le projet de construction du chenal du port de pêche de Grand-Lahou qui vise à assurer la navigabilité de l'estuaire afin de redonner une vitalité à l'économie de la région. Ainsi, les variations spatio-temporelles de quelques paramètres physico-chimiques et des indicateurs de pollution chimique ont été étudiées en relation avec les variations saisonnières des paramètres hydrologiques et l'instabilité de la passe de Grand-Lahou. La variation des paramètres physico-chimiques de la lagune de Grand-Lahou est fortement influencée par la dynamique du fleuve Bandama, des petites rivières côtières et du degré d'ouverture ou de fermeture de la passe. Ainsi, la turbidité reste très élevée aux environs de la passe pendant la crue du fleuve Bandama (70 NTU < turbidité < 120 NTU). Par ailleurs, la salinité reste élevée (18\%o en moyenne) en périodes d'étiages où les eaux marines repoussent les eaux continentales. En revanche, en périodes de hautes eaux du fleuve Bandama et des rivières côtières, la salinité diminue pour atteindre des valeurs allant de $0,05 \%$ à $10 \%$ o. Les teneurs en sels nutritifs $(\mathrm{CV}$ $>15 \%$ ) sont relativement élevées avec des baissent significatives en périodes d'étiage du fait de la prolifération des algues, de la photosynthèse, des activités bactériennes et de la sédimentation importante.

(C) 2013 International Formulae Group. All rights reserved

Mots clés: Hydrologie, hydrochimie, estuaire, passe, lagune de Grand-Lahou.

\section{INTRODUCTION}

Les estuaires, du fait des atouts écologiques, touristiques et économiques qu'ils présentent, subissent une pression anthropique importante conduisant à des perturbations du fonctionnement naturel de ces écosystèmes (Amiard-Triquet, 1989 ; Bryan et Langoston, 1992). En Côte d'Ivoire, du fait des aménagements littoraux et des variations du niveau de la mer ces dernières années, les estuaires connaissent d'énormes perturbations environnementales qui se traduisent par la pollution des eaux, la modification de leurs morphologies et le déplacement des embouchures. L'estuaire de Grand-Lahou, lieu de rencontre entre le fleuve Bandama, les rivières Boubo et Gô, le système lagunaire de Grand-Lahou et l'Océan Atlantique représente, de part sa diversité écologique et environnementale, une importante source de subsistance et une source de revenue pour les populations 
riveraines dont la principale activité économique est la pêche traditionnelle (Abé et al., 1993 ; Mama, 2011). Le système lagunaire de Grand-Lahou est constitué des lagunes Niouzoumou, Tadjo, Mackey et Tagba. La lagune Tagba est le seul exutoire en mer de ce système lagunaire. L'estuaire de GrandLahou, qui devrait être protégé du fait de son importance vitale pour les populations riveraines, subit de plus en plus une importante dynamique sédimentaire et une pollution des eaux due aux rejets domestiques (ordures ménagères et eaux usées) directement dans le système lagunaire et à la pratique de la pêche souvent avec des produits chimiques. Ce qui a pour conséquences la modification de la morphologie de l'estuaire et la migration de la passe d'Est en Ouest (Abé et al., 1996 ; Wognin et al., 2007), la variation significative des paramètres physico-chimiques, chimiques et microbiologiques des plans d'eau (Konan et al., 2008 ; konan et al., 2009 ; Tiekoura et al., 2010 ; Kouadio et al., 2011). Ainsi, les travaux de ces auteurs indiquent que :

- l'exutoire actuel du fleuve Bandama, qui se constitue d'une passe, ne cesse de migrer sous l'effet des variations saisonnières liées aux débits de ce cours d'eau, des marées et houles océaniques (Wognin et al., 2008) ;

- les tirants d'eau en lagune sont généralement faibles; ce qui rend la navigation en lagune, au niveau de l'embouchure assez difficile (Konan et al., 2008) ;

- la pêche qui occupait naguère jusqu'à $60 \%$ des activités de la population connaît une baisse et on assiste donc à une paupérisation plus accentuée de la population de Grand Lahou (Ecoutin et al., 1994) ;

- les évolutions des poissons migrateurs, les capacités de nurseries pour certains d'entre eux (aloses, crevettes, etc.) sont fortement perturbées. Un appauvrissement de la lagune, de plus en plus évoqué, est à craindre (Konan et al., 2009 ; Villanueva, 2004).

Dans le souci de redonner une vitalité à l'économie de la région, l'Etat ivoirien a construit une école de pêche à Grand-Lahou avec un port d'application et des bateaux pour les activités pratiques en eau continentale et en eau marine. Cependant, les bateaux du Lycée Professionnel de Grand-Lahou dont les objectifs de formation sont orientés vers les métiers de la pêche ne peuvent pas accéder à la mer parce que la passe reste innavigable. Pour permettre aux bateaux-écoles dans le cadre de la formation pratique des apprenants de relier le port et la mer, un projet de construction d'un chenal reliant le port d'application du Lycée Professionnel de Grand-Lahou à la mer est envisagé et doit rentrer dans sa phase d'exécution.

Ce canal, bien qu'il permettra de résoudre des problèmes socio-économiques dont souffre la région de Grand-Lahou, pourrait avoir des impacts environnementaux importants sur l'estuaire de Grand-Lahou.

Dans le souci d'accompagner le projet de construction du canal de Grand-Lahou, cette étude se propose de caractériser l'état actuel du système lagunaire de Grand-Lahou et de faire des propositions pour la préservation de cet écosystème.

\section{MATERIEL ET METHODES Site d'étude}

La zone estuarienne de Grand-Lahou est située d'une part entre les $4^{\circ} 26$ et $5^{\circ} 20$ de latitude Nord et d'autre part entre $4^{\circ} 20$ et $5^{\circ} 20$ de longitude Ouest (Figure 1). La passe de Grand-Lahou constitue l'unique débouché en mer du système lagunaire, du fleuve Bandama et de la rivière Boubo. Le climat de la région de Grand-Lahou est de type équatorial caractérisé par une grande saison sèche de décembre à mars, une grande saison des pluies d'avril à juillet, une petite saison sèche d'août à septembre (période d'upwelling) et une petite saison des pluies d'octobre à novembre. L'hydrologie de l'estuaire est essentiellement sous l'influence des régimes du fleuve Bandama dominés par le régime tropical de transition du nord du bassin versant du fait de sa forme allongée, des cours d'eau côtiers (régime forestier) et des saisons marines. 


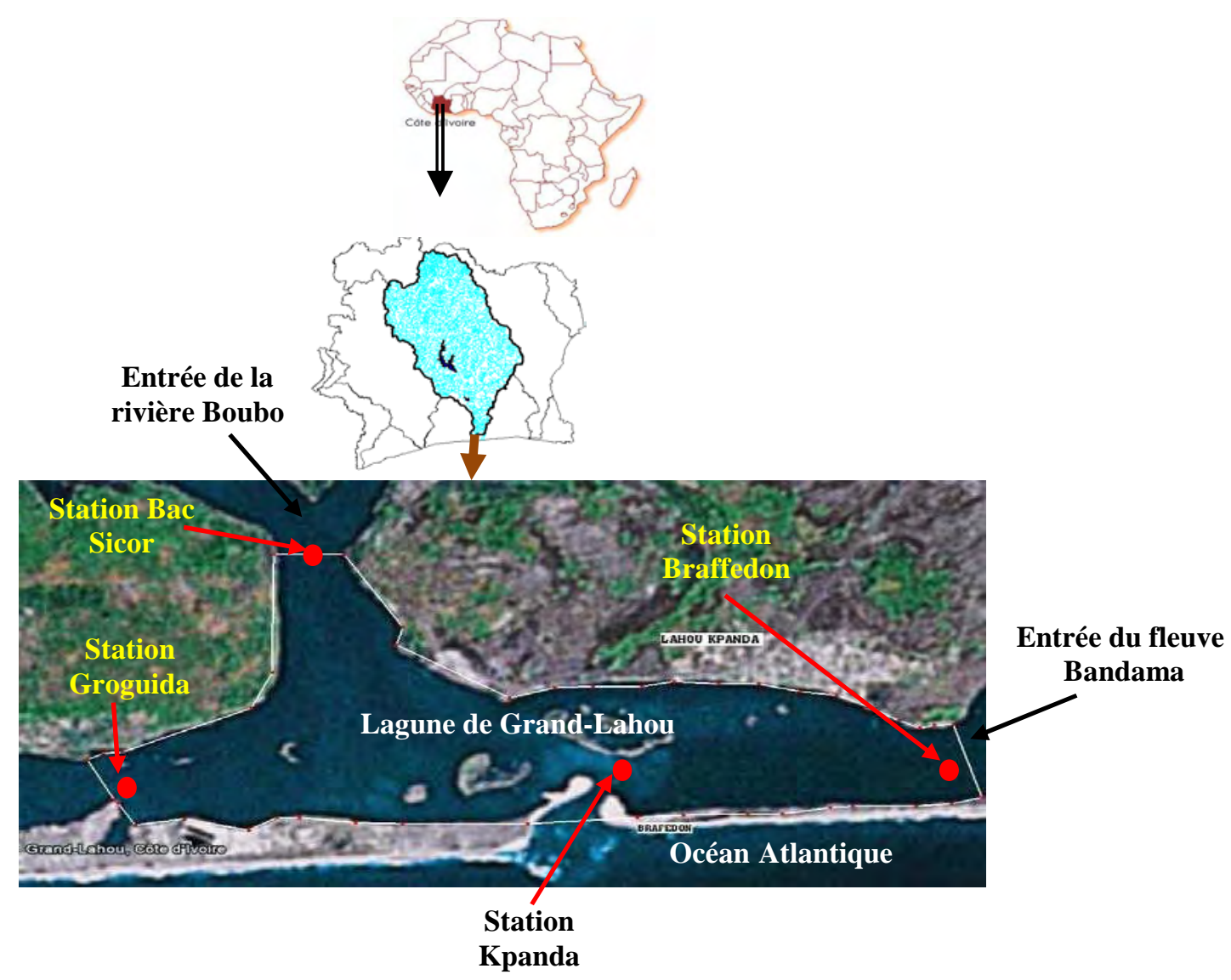

Figure 1: Localisation de la zone estuarienne de Grand-Lahou.

Méthode

Collecte des données et des échantillons

Données hydrologiques

Les caractéristiques hydrologiques (débits) du fleuve Bandama et de la rivière Boubo ont été obtenues auprès de la Direction de l'Eau du Ministère des Infrastructures Economiques de la Côte d'Ivoire. Les données pluviométriques de la ville de Grand-Lahou ont été acquises auprès de la Société d'Exploitation et de Développement Aéroportuaire, Aéronautique et Météorologique (SODEXAM).

\section{Echantillonnage}

Les caractéristiques physico-chimiques de la lagune ont été étudiées à travers douze (12) campagnes de mesures effectuées mensuellement de janvier 2010 à décembre
2010. Les campagnes de mesures et d'échantillonnage ont été réalisées sur quatre (4) stations où s'effectuent les transferts des différentes masses d'eau qui alimentent la zone estuarienne de Grand-Lahou (Figure 1). Les échantillons prélevés ont été soigneusement conservés à basse température $\left(\mathrm{T}^{\circ} \leq 4{ }^{\circ} \mathrm{C}\right)$, dans une glacière et ont été analysés au laboratoire.

Analyses physico-chimiques et détermination des sels nutritifs

La température, la salinité, le $\mathrm{pH}$, l'oxygène dissous et la turbidité ont été mesurés in situ à l'aide d'une sonde multiparamètre HANNA 9828.

Les nitrates et les nitrites ont été dosés selon les méthodes normalisées d'AFNOR : NFT 90013/90012 (AFNOR, 1994). Le 
dosage des ions phosphates a été réalisé selon la méthode spectrophotométrie d'adsorption moléculaire (Aminot et Chaussepied, 1983).

La corrélation paramétrique de Bravais Pearson (Artusi et al., 2002) et la classification ascendante hiérarchique ont été utilisées pour le traitement des données. Ces méthodes statistiques permettent une explication de l'évolution de la qualité des eaux en fonction de la variation des indicateurs de pollution. Elles permettent également d'apprécier si chaque phénomène observé est suffisamment exprimé et que la somme cumulée de la contribution des principaux facteurs considérés est d'environ $70 \%$.

\section{RESULTATS}

\section{Caractéristiques hydrologiques de l'estuaire de Grand-Lahou}

Les variations saisonnières des débits des principaux affluents qui alimentent l'estuaire de Grand-Lahou montrent une grande différence entre les écoulements du fleuve Bandama (Figure 2a) dont le régime ne suit pas celui de la pluviométrie de GrandLahou et celui des rivières côtières (Figure 2b) qui ont une évolution similaire à celle des pluies de la région. Ainsi, la rivière Boubo connaît deux crues annuelles (juin et octobre) tandis que le fleuve Bandama enregistre une seule crue annuelle en octobre. Les variations saisonnières de ces débits ont montré que l'écart entre les débits d'étiage et les débits de crue est important. En effet, les débits du fleuve Bandama varient entre $58 \mathrm{~m}^{3} / \mathrm{s}$ en période d'étiage (février) à $765 \mathrm{~m}^{3} / \mathrm{s}$ en période de crue (octobre). Au niveau de la rivière Boubo, les débits varient entre $0,6 \mathrm{~m}^{3} / \mathrm{s}$ pendant la grande saison sèche (février) à 27 $\mathrm{m}^{3} / \mathrm{s}$ pendant la grande saison des pluies (juin).

$\mathrm{Au}$ niveau de la pluviométrie de la région, les précipitations mensuelles enregistrées varient entre $17 \mathrm{~mm}$ de pluie au mois de janvier et $445 \mathrm{~mm}$ au mois de juin (Figure 3). Ainsi, sur un total d'environ $1460 \mathrm{~mm}$ de pluie par an, la grande saison des pluies enregistre plus de $64 \%$ des précipitations contre $17 \%$ pour la petite saison des pluies. Par ailleurs, il est tombé $188 \mathrm{~mm}$ de pluie pendant la grande saison sèche $(13 \%)$ et $89 \mathrm{~mm}$ pendant la petite saison sèche $(6 \%)$.

En ce qui concerne les intrusions marines, elles se font au niveau de la passe qui est fortement perturbée par l'érosion marine et les différents courants. L'estuaire de Grand-Lahou subit de plus en plus une importante dynamique sédimentaire qui entraîne constamment la modification de sa morphologie et sa migration (Figure 4).

\section{Caractéristiques physico-chimiques des eaux de l'estuaire de Grand-Lahou}

Le Tableau 1 et la Figure 5 permet d'apprécier les variations des paramètres physico-chimiques dans les eaux de l'estuaire de Grand-Lahou. Dans l'ensemble, les variations saisonnières des paramètres étudiés sont plus significatives que les variations spatiales (Figure 5).

En ce qui concerne la température, elle varie entre $17,36{ }^{\circ} \mathrm{C}$ pendant la période d'upwelling et $30,84^{\circ} \mathrm{C}$ en grande saison sèche avec une température moyenne de $28^{\circ} \mathrm{C}$ (Figure 5a). Sur le plan spatial, la température est relativement homogène dans la lagune $(\mathrm{CV}=7 \%)$ sauf aux environs de la passe et au débouché du fleuve Bandama (station Kpanda et Braffedon) où sont enregistrées les plus basses pendant la période d'upwelling. $\mathrm{Au}$ niveau de la salinité, on observe à la fois des variations saisonnières et spatiales importantes. Elle varie entre $0,05 \%$ pendant la petite saison des pluies qui coïncide avec la période de crue du fleuve Bandama et $32,7 \%$ o au cours de la petite saison sèche qui correspond à la période d'upwelling (Figure 5b). Au plan spatial, la salinité varie significativement d'une zone à une autre avec un coefficient de variation de l'ordre de $53 \%$. Les plus faibles valeurs de la salinité s'observent au débouché du fleuve Bandama (station Braffedon) et les valeurs les plus élevées à l'Ouest de l'estuaire (station Groguida). 
Quant à la teneur en oxygène dissous, elle varie de $0,2 \mathrm{mg} / \mathrm{L}$ à $25,87 \mathrm{mg} / \mathrm{L}$ respectivement pendant la grande saison sèche et la grande saison des pluies (Figure 5c). Les coefficients de variation de l'oxygène dissous élevés $(33 \%<\mathrm{CV}<88 \%)$ montrent qu'il y a une grande variabilité entre les eaux en saison des pluies et en saison sèche.

Pour ce qui est du pH, il est globalement compris entre 6,60 au cours de la grande saison des pluies et 8,46 pendant la grande saison sèche. Au plan spatial, sur toute l'année, les variations sont faibles $(\mathrm{CV}=8 \%)$ dans toute la zone estuarienne (Figure $5 \mathrm{~d}$ ).

Les valeurs moyennes mensuelles de la turbidité sont comprises entre 32,22 NTU pendant la grande saison sèche et 120,70 NTU au cours de la petite saison des pluies qui coïncide avec la crue du fleuve Bandama (Figure 5e). Au plan spatial, la turbidité est plus élevée dans la passe et la zone de pénétration du fleuve Bandama (Station Kpanda et Braffedon). Les coefficients de variation de $4 \%$ et $11 \%$ observés respectivement à la station Groguida et Bac Sicor sont faibles pendant que ceux des stations Kpanda et Braffedon restent élevés avec des valeurs respectives de $58 \%$ et $67 \%$.

\section{Paramètres chimiques}

Le Tableau 2 et la Figure 6 présentent les variations saisonnières des sels nutritifs dans les eaux de l'estuaire de la lagune de Grand-Lahou. Les teneurs moyennes saisonnières en phosphates $\left(\mathrm{PO}_{4}{ }^{3-}\right)$, nitrates $\left(\mathrm{NO}_{3}{ }^{-}\right)$et nitrites $\left(\mathrm{NO}_{2}{ }^{-}\right)$varient respectivement entre $0,05 \mathrm{mg} / \mathrm{L}$ (petite et grande saison sèche) et $0,40 \mathrm{mg} / \mathrm{L}$ (petite saison des pluies) ; 0,002 $\mathrm{mg} / \mathrm{L}$ (grande saison sèche) et $0,83 \mathrm{mg} / \mathrm{L}$ (petite saison sèche) et entre $0,03 \mathrm{mg} / \mathrm{L}$ (grande saison sèche) et $0,79 \mathrm{mg} / \mathrm{L}$ (grande saison des pluies). Les coefficients de variation en sels nutritifs (35\% < CV des $\mathrm{PO}_{4}{ }^{3-} \leq 96 \% ; 68 \%<\mathrm{CV}$ des $\mathrm{NO}_{3}{ }^{-} \leq 101 \% ; \quad 73 \%<\mathrm{CV}$ des $\mathrm{NO}_{2}{ }^{-} \leq 99 \%$ ) sont élevés et supérieurs à $15 \%$.
Corrélation entre les paramètres physicochimiques et chimiques

L'analyse des données suivant la méthode de la classification ascendante hiérarchique et le coefficient de corrélation de Pearson avec le type de similarité a permis de procéder au regroupement entre l'ensemble des paramètres hydrochimiques étudiés d'une part, et, d'autre part, entre les stations en fonction de leur similarité dans la variation de ces paramètres hydrochimiques.

Ainsi, deux groupes peuvent être distingués au niveau des stations de la zone estuarienne de la lagune de Grand-Lahou, comme indiqué à la Figure 7. Le premier groupe comprend les stations Bac Sicor et Groguida qui sont un peu plus éloignées de l'embouchure du fleuve Bandama et le second groupe comprend des stations Kpanda (à proximité de la passe) et Braffedon (embouchure du fleuve Bandama).

De même, au niveau des paramètres hydrochimiques, il y a également deux groupes: un premier groupe formé par les paramètres physico-chimiques (température, salinité, oxygène dissous, $\mathrm{pH}$ et Turbidité) et un second, formé par les paramètres chimiques $\left(\mathrm{PO}_{4}{ }^{3}, \mathrm{NO}_{3}{ }^{-}\right.$et $\left.\mathrm{NO}_{2}{ }^{-}\right)$.

L'analyse de corrélation de Bravais Pearson effectuée entre les différents paramètres hydrochimiques est présentée dans le Tableau 3. Cette analyse montre que le paramètre salinité est corrélé positivement avec le $\mathrm{pH}(0,72)$ et négativement avec la turbidité $(-0,95)$, les nitrates $(-0,91)$ et les nitrites $(-0,86)$. Par ailleurs, la température est corrélée négativement avec la turbidité $(-0,56)$ et les sels nutritifs $(-0,86)$ avec les phosphates. Ce tableau indique également que l'oxygène dissous est corrélé positivement avec les facteurs $\mathrm{pH}(0,67)$ et négativement avec les phosphates $(-0,68)$ et les nitrates $(-0,66)$. Enfin, il existe une forte corrélation positive entre la turbidité et les sels nutritifs $(0,65$ avec les phosphates, 0,97 avec nitrates et 0,74 avec les nitrites). 
Tableau 1: Caractéristiques des paramètres physico-chimiques des eaux de l'estuaire de GrandLahou.

\begin{tabular}{|c|c|c|c|c|c|c|}
\hline Stations & & $\begin{array}{c}\text { Température } \\
\left({ }^{\circ} \mathbf{C}\right)\end{array}$ & $\begin{array}{c}\text { Salinité } \\
(\% \circ)\end{array}$ & $\begin{array}{c}\text { Oxygène dissous } \\
(\mathrm{mg} / \mathrm{L})\end{array}$ & $\mathbf{p H}$ & $\begin{array}{c}\text { Turbidité } \\
\text { (NTU) }\end{array}$ \\
\hline \multicolumn{7}{|l|}{ Bac Sicor } \\
\hline & Minimum & 20,73 & 1,19 & 2,3 & 6,62 & 39,35 \\
\hline & Maximum & 30,84 & 23,44 & 25,87 & 8,39 & 53,3 \\
\hline & Moyenne & 27,50 & 12,87 & 7,08 & 7,75 & 44,26 \\
\hline & Ecart Type & 2,83 & 8,85 & 6,23 & 0,60 & 5,02 \\
\hline & $\mathrm{CV}(\%)$ & 10 & 69 & 88 & 8 & 11 \\
\hline \multicolumn{7}{|l|}{ Groguida } \\
\hline & Minimum & 25,72 & 10,23 & 0,7 & 6,58 & 34,8 \\
\hline & Maximum & 30,16 & 24,75 & 19,77 & 8,33 & 39,4 \\
\hline & Moyenne & 28,32 & 18,95 & 5,75 & 7,74 & 36,51 \\
\hline & Ecart Type & 1,29 & 5,66 & 4,83 & 0,66 & 1,39 \\
\hline & $\mathrm{CV}(\%)$ & 5 & 30 & 84 & 9 & 4 \\
\hline \multicolumn{7}{|l|}{ Kpanda } \\
\hline & Minimum & 17,36 & 0,95 & 0,9 & 6,63 & 32,20 \\
\hline & Maximum & 28,14 & 32,70 & 7,275 & 8,49 & 107,3 \\
\hline & Moyenne & 28,32 & 18,95 & 5,75 & 7,74 & 36,51 \\
\hline & Ecart Type & 2,87 & 11,36 & 1,90 & 0,61 & 21,22 \\
\hline & $\mathrm{CV}(\%)$ & 10 & 60 & 33 & 8 & 58 \\
\hline \multicolumn{7}{|l|}{ Braffedon } \\
\hline & Minimum & 26,30 & 0,05 & 0,20 & 6,6 & 36,4 \\
\hline & Maximum & 27,68 & 29,71 & 6,73 & 8,28 & 120,70 \\
\hline & Moyenne & 28,32 & 18,95 & 5,75 & 7,74 & 36,51 \\
\hline & Ecart Type & 0,74 & 11,29 & 1,95 & 0,62 & 24,52 \\
\hline & $\mathrm{CV}(\%)$ & 3 & 60 & 34 & 8 & 67 \\
\hline
\end{tabular}

$\mathrm{CV}$ : Coefficient de variation.

Tableau 2: Caractéristiques des paramètres chimiques des eaux de l'estuaire de Grand-Lahou.

\begin{tabular}{llccc}
\hline Stations & Minimum & $\begin{array}{c}\text { Phosphates } \\
(\mathbf{m g} / \mathbf{L})\end{array}$ & $\begin{array}{c}\text { Nitrates } \\
(\mathbf{m g} / \mathbf{L})\end{array}$ & $\begin{array}{c}\text { Nitrites } \\
(\mathbf{m g} / \mathbf{L})\end{array}$ \\
\hline Bac Sicor & 0,05 & 0,05 & 0,06 \\
& Maximum & 0,25 & 0,26 & 0,62 \\
& Moyenne & 0,16 & 0,13 & 0,38 \\
& Ecart Type & 0,08 & 0,09 & 0,27 \\
& CV (\%) & 51 & 68 & 73 \\
\hline Groguida & Minimum & 0,05 & & \\
& Maximum & 0,40 & 0,01 & 0,03 \\
& Moyenne & 0,17 & 0,12 & 0,52 \\
& Ecart Type & 0,16 & 0,07 & 0,25 \\
& CV (\%) & 96 & 0,05 & 0,21 \\
& & 824 & 73 & 84 \\
\hline & & & &
\end{tabular}




\begin{tabular}{llccc}
\hline Kpanda & Minimum & 0,14 & 0,00 & 0,12 \\
& Maximum & 0,40 & 0,52 & 0,79 \\
& Moyenne & 0,25 & 0,23 & 0,32 \\
& Ecart Type & 0,12 & 0,22 & 0,31 \\
& CV $(\%)$ & 49 & 96 & 99 \\
\hline Braffedon & & & & \\
& Minimum & 0,13 & 0,00 & 0,04 \\
& Maximum & 0,35 & 0,83 & 0,68 \\
& Moyenne & 0,27 & 0,35 & 0,37 \\
& Ecart Type & 0,10 & 0,35 & 0,35 \\
& CV $(\%)$ & 35 & 101 & 96 \\
\hline CV:Coefficient de variation & & &
\end{tabular}

Tableau 3: Matrice de corrélation (Bravais Pearson) entre les paramètres physico-chimiques et les indicateurs de la pollution chimique des eaux de la lagune de Grand-Lahou.

\begin{tabular}{lcccccccc}
\hline Variables & Temp. & Sal. & $\mathbf{O}_{2}$ & $\mathbf{p H}$ & Turb. & $\mathbf{P O}_{4}{ }^{3-}$ & $\mathbf{N O}_{3}{ }^{-}$ & $\mathbf{N O}_{2}{ }^{-}$ \\
\hline Temp. & 1 & & & & & & & \\
Sal. & 0,32 & 1 & & & & & & \\
$\mathrm{O}_{2}$ & 0,22 & 0,32 & 1 & & & & & \\
$\mathrm{pH}$ & $-0,18$ & $\mathbf{0 , 7 3}$ & $\mathbf{0 , 6 7}$ & 1 & & & & \\
Turb. & $\mathbf{- 0 , 5 6}$ & $\mathbf{- 0 , 9 5}$ & $-0,49$ & $\mathbf{- 0 , 6 8}$ & 1 & & & \\
$\mathrm{PO}_{4}{ }^{3-}$ & $\mathbf{- 0 , 8 6}$ & $-0,37$ & $\mathbf{- 0 , 6 8}$ & $-0,19$ & $\mathbf{0 , 6 5}$ & 1 & & \\
$\mathrm{NO}_{3}{ }^{-}$ & $-0,46$ & $\mathbf{- 0 , 9 1}$ & $\mathbf{- 0 , 6 6}$ & $\mathbf{- 0 , 7 8}$ & $\mathbf{0 , 9 7}$ & $\mathbf{0 , 6 6}$ & 1 & \\
$\mathrm{NO}_{2}{ }^{-}$ & $-0,29$ & $\mathbf{- 0 , 8 6}$ & 0,20 & $-0,36$ & $\mathbf{0 , 7 4}$ & 0,08 & $\mathbf{0 , 6 0}$ & 1 \\
\hline \multicolumn{7}{c}{ Les valeurs en gras sont significativement différentes de 0 à un niveau de signification alpha $=0,05}$. & &
\end{tabular}
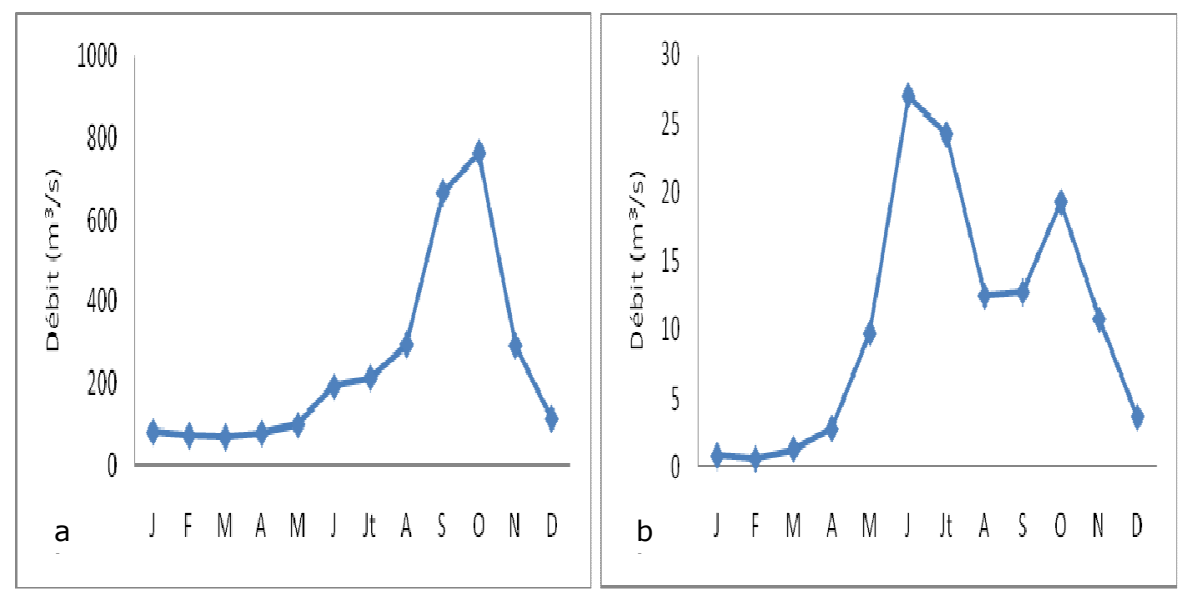

Figure 2: Variation des valeurs moyennes mensuelles du débit: (a) du fleuve Bandama et (b) de la rivière Boubo de 2000 à 2010. 


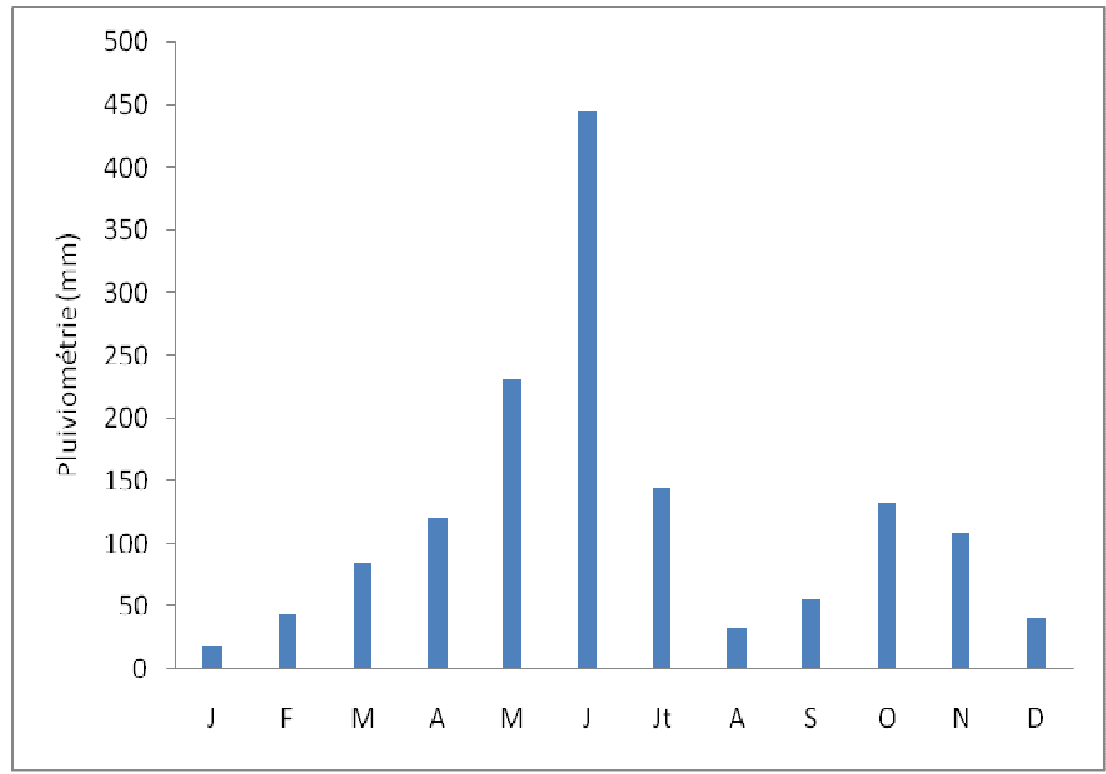

Figure 3: Précipitations moyennes mensuelles de Grand-Lahou de 2000 à 2010.
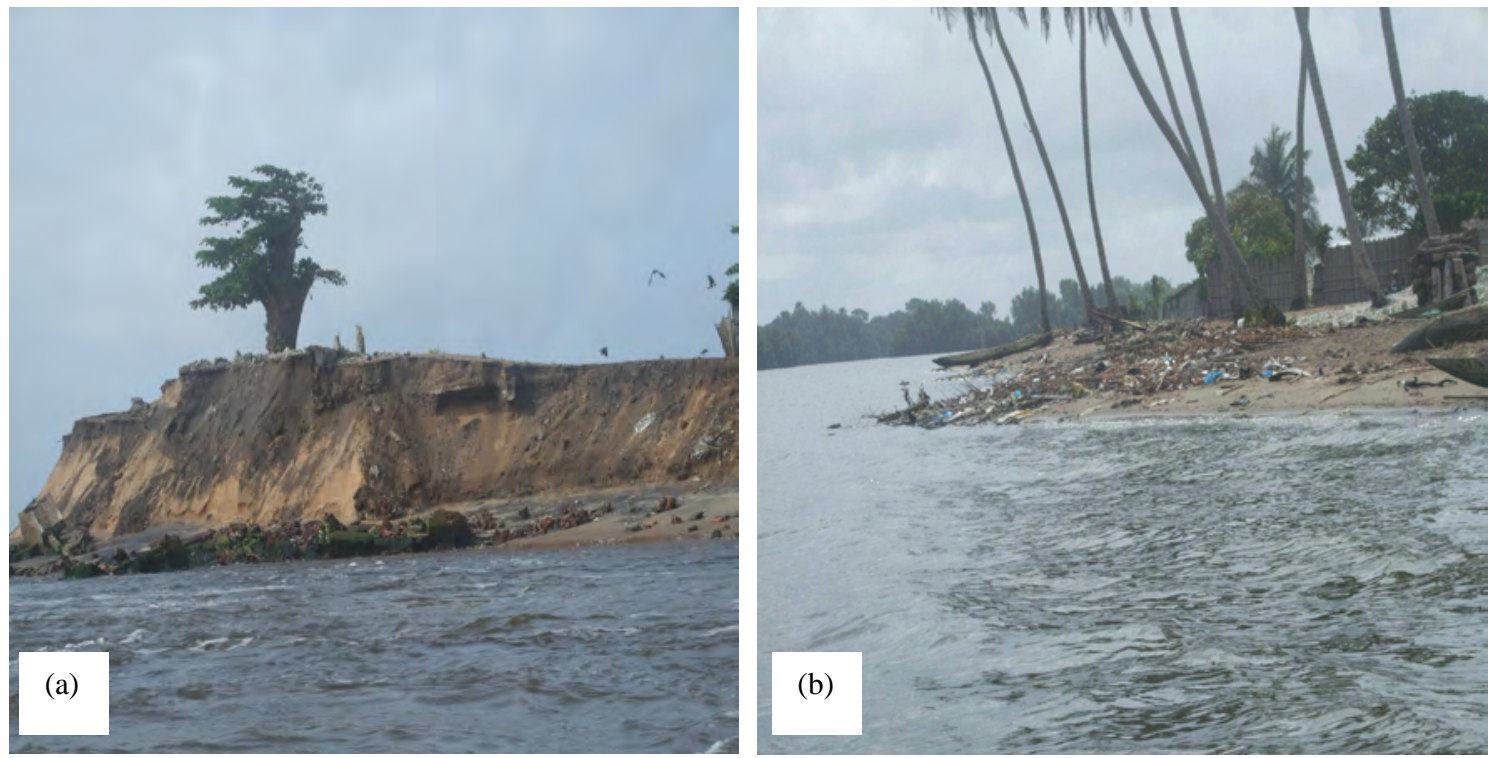

Figure 4: Dynamique sédimentaire : (a) Passe (Lahou Kpanda) et (b) Estuaire (Ile Ségui). 
(a)

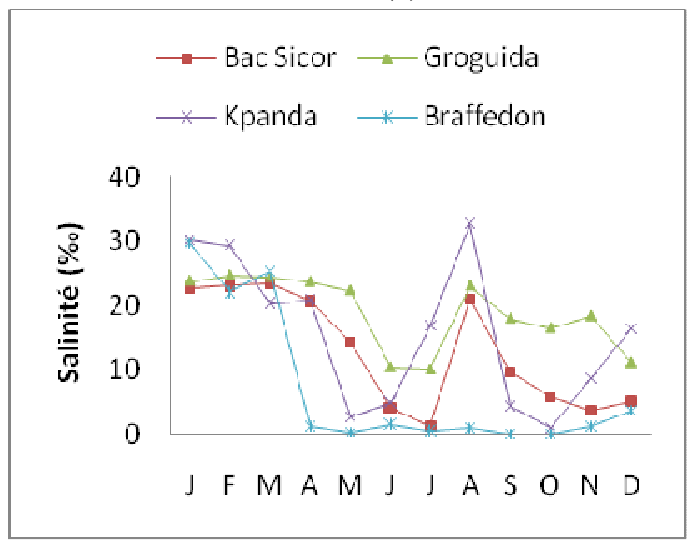

(c)

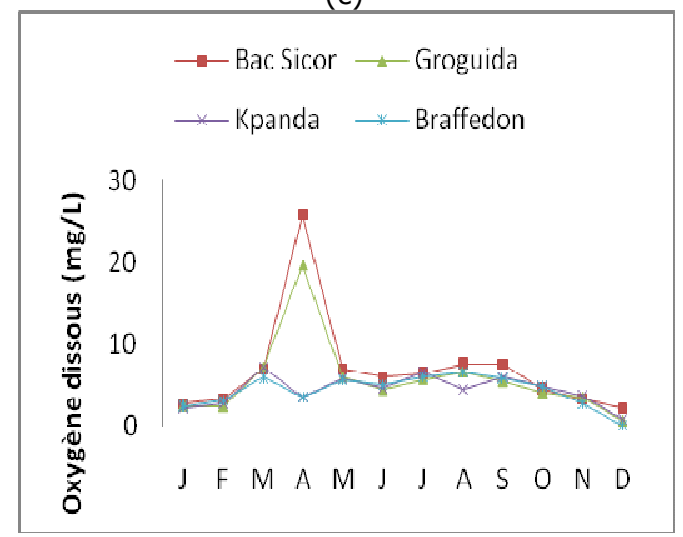

(b)

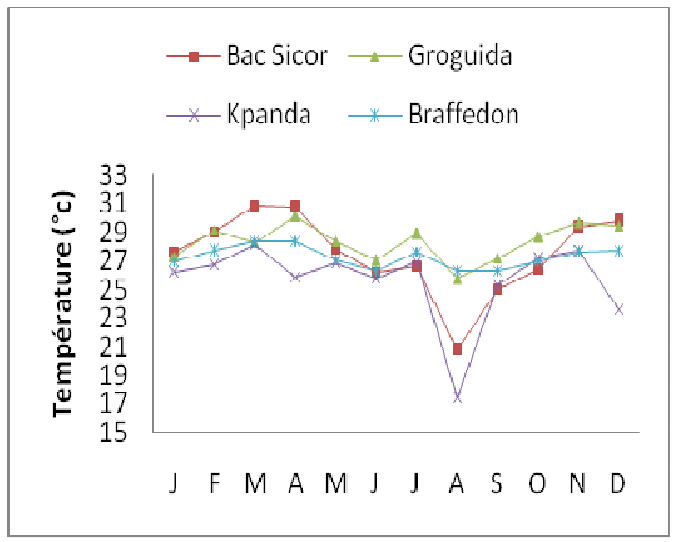

(d)

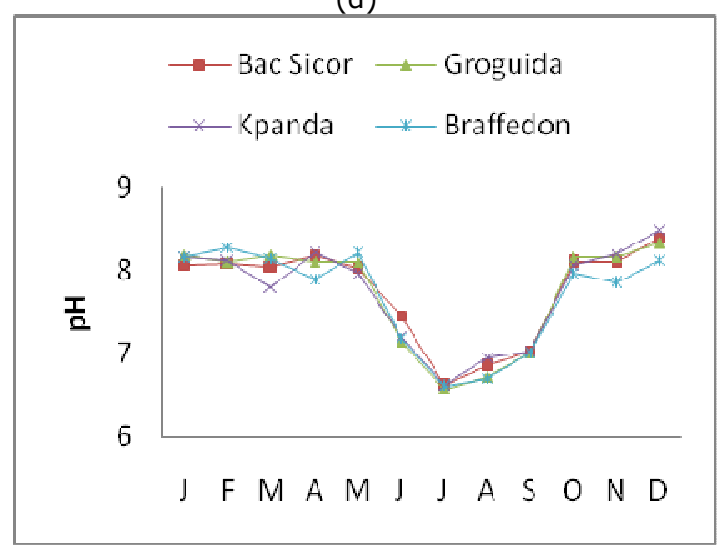

(c)

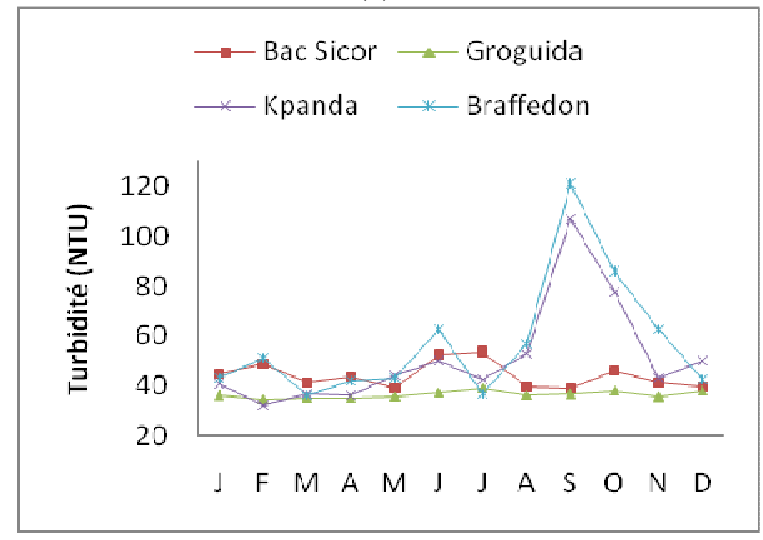

Figure 5: Variation des valeurs moyennes mensuelles des paramètres physico-chimiques des eaux de la zone estuarienne de la lagune de Grand-Lahou: (a) température, (b) salinité), (c) oxygène dissous, (d) $\mathrm{pH}$, (e) turbidité. 
(a)

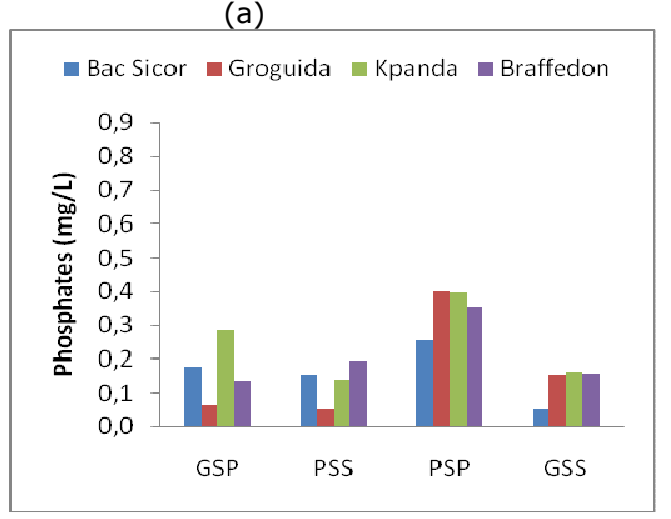

(b)

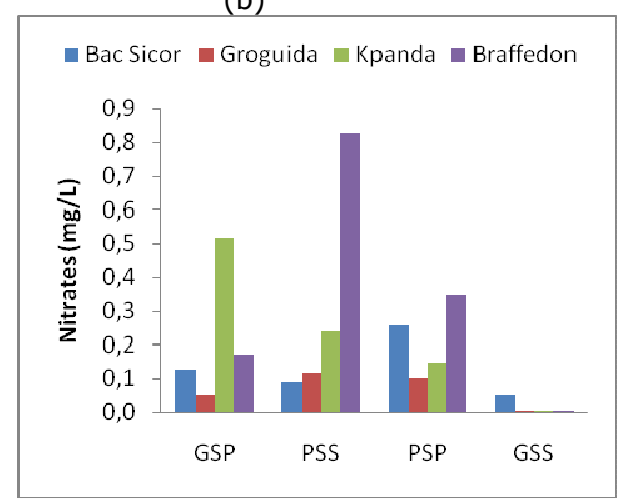

(c)

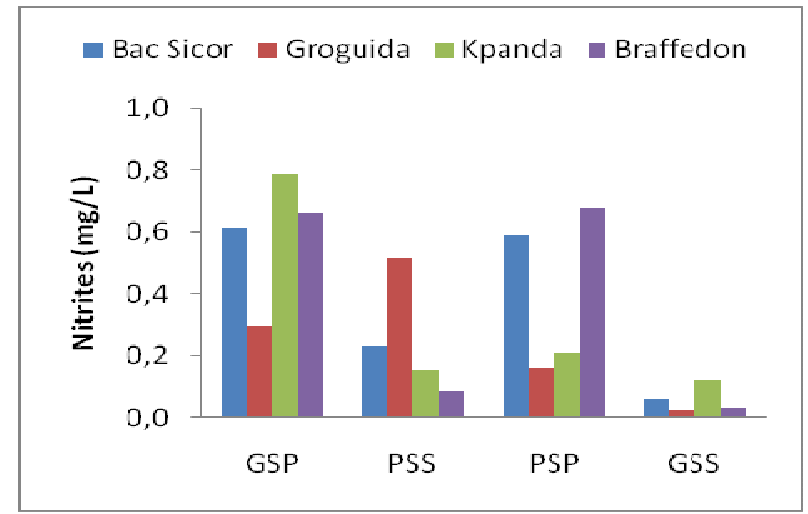

Figure 6: Variation saisonnière des concentrations moyennes en sels nutritifs des eaux de la zone estuarienne de la lagune de Grand-Lahou : (a) Phosphates, (b) Nitrates, (c) Nitrites. GSP : Grande Saison des Pluies, PSS : Petite Saison Sèche, PSP : Petite Saison des Pluies, GSS : Grande Saison Sèche.

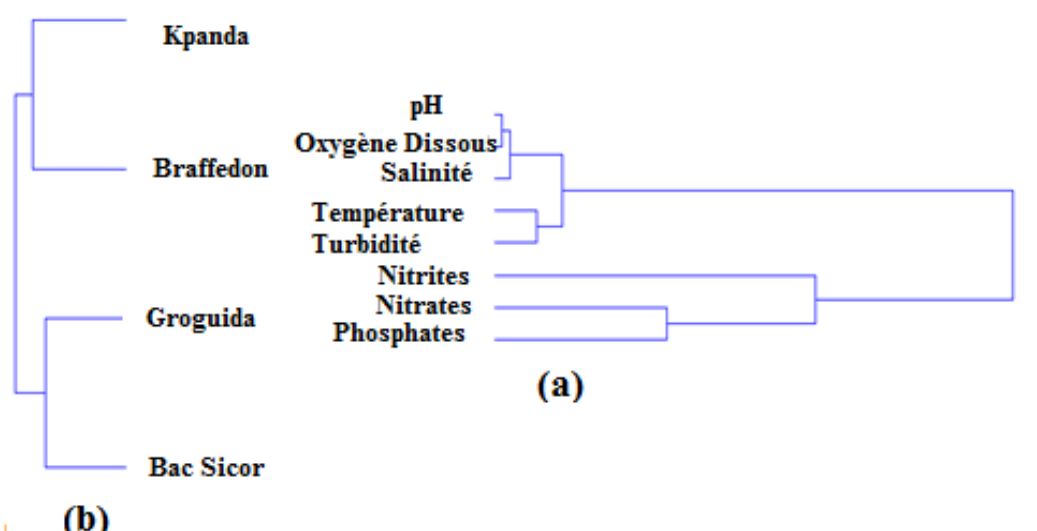

Figure 7: Dendrogramme de la répartition des paramètres hydrochimiques (a) des eaux de la lagune de Grand-Lahou en fonction des stations de mesure (b). 


\section{DISCUSSION}

L'estuaire de Grand-Lahou, qui représente une importante source de subsistance et une source de revenue pour les populations riveraines dont la principale activité économique est la pêche traditionnelle, est de plus en plus menacée par des phénomènes sédimentaires (Abé et al., 1996 ; Yao et al., 2010) et une pollution des eaux lagunaires (konan et al., 2009 ; Tiekoura et al., 2010; Kouadio et al., 2011). Les travaux de Wognin et al. (2007, 2008) ont montré que l'estuaire de Grand-Lahou subit d'importantes modifications qui se traduisent par sa migration d'Est en Ouest et des évolutions de la morphologie du fond sous l'effet de la dynamique hydrologique. La circulation des masses d'eau dans l'estuaire et les activités anthropiques sur le bassin versant du Bandama et des rivières côtières ont une influence sur les paramètres physicochimiques.

La température et le $\mathrm{pH}$ des eaux de la zone estuarienne de Grand-Lahou ont une distribution homogène $(\mathrm{CV}<15 \%)$ dans l'ensemble des stations de mesure. Cette homogénéité pourrait s'expliquer par la faible profondeur de cette lagune qui favorise le mélange des eaux de surface et du fond. Ce constat semble être une particularité des lagunes tropicales car, de faibles variabilités spatiales de la température et du $\mathrm{pH}$ ont été également observés dans les eaux estuariennes d'autres lagunes tropicales par Kouassi et al. (2005), Villanueva (2004) et Konan et al. (2008). Par ailleurs, les variations de ces deux paramètres en fonction des saisons sont aussi relativement faibles. Selon Dufour (1984), Allenbach et al. (2010), les variations de la température dans les estuaires ont pour origine les milieux frontaliers.

Contrairement à la température et au $\mathrm{pH}$, la salinité, la turbidité et l'oxygène dissous varient sensiblement avec des fluctuations liées aux saisons et aux différents apports. La salinité des eaux de la lagune de Grand-Lahou est contrôlée par les apports d'eau douce du fleuve Bandama et de la rivière Boubo, les précipitations directes et les marées via la passe de Grand-Lahou. Au plan de la dynamique hydrologique, l'évolution des valeurs moyennes mensuelles des débits et de la pluviométrie montre qu'au cours des mois de septembre et octobre, en dehors de la station Groguida, les paramètres physicochimiques (salinité, turbidité et oxygène dissous) sont essentiellement influencés par les eaux du fleuve Bandama. Ces résultats sont conformes à ceux observés par (Wognin et al., 2008) et Konan et al. (2008) au cours de la période allant de 2003 à 2006. Wognin et al. (2008) ont montré que les examens des variations de la turbidité et des teneurs en matières en suspension dans l'estuaire du Bandama sont fortement liés à la circulation des masses d'eau qui alimentent ce secteur. Ces auteurs ont fait apparaître une forte corrélation entre le régime hydrologique, la turbidité et la salinité.

La construction du chenal du port de pêche de Grand-Lahou entraînera une remise en suspension des sédiments, donc une augmentation de la turbidité et de la concentration en matières en suspension. Ce qui peut affecter la qualité chimique et biologique de l'estuaire. Pour ce faire, une étude d'impact et des mesures d'atténuation des impacts de ce projet s'avèrent nécessaires pour sauver et protéger cet écosystème (Mohamed et al., 2008).

Les teneurs en sels nutritifs (phosphates, nitrates et nitrites) des eaux de la zone estuarienne de Grand-Lahou sont relativement élevées au cours des saisons des pluies et de crues. En effet, au cours de ces périodes le drainage des eaux de ruissellement, des bassins versants sont à l'origine des apports anthropiques en sels nutritifs (Millet, 1986) et du relargage des sédiments dans la lagune (Lefebvre et Cordier, 2010). Cette situation pourrait s'expliquer par le fait qu'en Côte d'Ivoire, les engrais agricoles sont épandus généralement entre mars et avril. Ces derniers sont entraînés par les précipitations qui « lessivent » (Dorioz et al., 2004) puis sont drainés par les eaux de 
ruissellement jusqu'aux lits des affluents (fleuve Bandama, les rivières Boubo et Gô). Par ailleurs, la circulation de ces eaux en saison des pluies et le renouvellement des eaux entraînent une remontée des sédiments qui viennent également enrichir la colonne d'eau. A ces phénomènes s'ajoute le fait que les populations riveraines et les animaux défèquent directement ou indirectement dans les eaux.

\section{Conclusion}

L'analyse des paramètres physicochimiques et des teneurs en sels nutritifs mesurés fait ressortir une variation significative de la qualité des eaux de l'estuaire de Grand-Lahou. En effet, la température, la salinité, l'oxygène dissous, le $\mathrm{pH}$ et la turbidité de l'eau de la zone estuarienne varient en fonction des apports du fleuve Bandama, des rivières forestières (Boubo et Gô) et des intrusions marines et de la variation de la morphologie de la passe. En période de crue ou de pluie, la largeur et la profondeur de cette passe sont élevées et les échanges entre la mer et la lagune sont plus importants. La mise en œuvre du projet de construction du chenal du port de pêche de Grand-Lahou aura un impact certain sur l'hydrologie et l'hydrochimie des eaux de l'estuaire de cette lagune. Pour ce faire, une étude d'impact et des mesures d'atténuation des impacts de ce projet s'avèrent nécessaires pour sauver et protéger cet écosystème, afin de préserver ses potentialités bioécologiques et améliorer les conditions socio-économique de la population riveraine.

\section{REMERCIEMENTS}

Cette recherche a fait l'objet d'un soutien financier et logistique de la Fondation Internationale pour la Science (IFS, Suède) et du Lycée professionnel de Grand-Lahou. Nous leur adressons nos sincères remerciements pour leur contribution à nos travaux.

\section{REFERENCES}

Abé J. 1993. Morphologie et hydrodynamique à l'embouchure du fleuve Bandama. Journal Ivoirien d'Océanologie et Limnologie, 2(2): 9-24.

Abe J, Bakayoro S, Bamba S, Cissoko S. 1996. L'hydrologie de l'estuaire du Comoé à Grand-Bassam (Côte d'Ivoire). Agronomie Africaine. Journal Ivoirien d'Océanologie et Limnologie, 8(3): 201212.

Adopo KL, Kouassi KL, Wognin AV, Monde S, Aka K. 2008. Caractérisation des sédiments et morphologie de l'embouchure du fleuve Comoé (GrandBassam, Côte d'Ivoire). Revue Paralia, 1: 2.1-2.10.

AFNOR. 1994. Qualité de l'Eau: Environnement, ( ${ }^{\text {ère }}$ edn). Association Française de Normalisation: Paris, France; 861.

Allenbach M, Mangeas M, Touraivane. 2010. Le suivi de la turbidité associée au dragage de Vavouto à proximité des sites néocalédoniens classés par l'Unesco. Revue Paralia, 3 : 7.1-7.12.

Amiard-Triquet C. 1989. Bioaccumulation et nocivité relatives de quelques polluants métalliques à l'égard des espèces marines. Bull. Ecol., 20: 129-151.

Aminot A, Chaussepied M. 1983. Manuel des Analyses Chimiques en Milieu Marin. Edition Jouve, Brest cnexo/bndo; 479.

Artusi R, Verderio P, Marubini E. 2002. Bravais-Pearson and Spearman correlation coefficients: meaning, test of hypothesis and confidence interval. The International Journal of Biological Markers, 17(2): 148-151.

Bryan GW, Langoston WJ. 1992. Bioavaibility, accumulation and effects heavy metals in sediments with special reference to united estuary. Environ. Pollution, 76: 89-131.

Dorioz JM, Quetin PLazarotte, JA, Bosse JP, Moreille JP. 2004. Bilan du phosphore dans un bassin versant du lac Léman: Conséquences pour la détermination de 
l'origine des flux exportés. Rev. Sci. Eau, 17(3): 329-354.

Dufour P. 1984. Production primaire d'une lagune tropicale (Ebrié, côte d'ivoire), Facteurs naturels et anthropiques. Thèse unique de Doctorat. Université Pierre et marie-curie, paris, p. 164.

Ecoutin JM, Durand JR, Lae R, Hié JP. 1994. L'exploitation des stocks. In Environnement des Ressources Aquatiques de Côte d'Ivoire, les Milieux Lagunaires (Tome II); 339-444.

Konan KS, Kouassi AM, Adingra AA, Dongui BK, Gnakri D. 2008. Variations saisonnières des paramètres abiotiques des eaux d'une lagune tropicale : la lagune de Grand-Lahou, Cote d'Ivoire. European Journal of Scientific research, 21(3): 376-393.

Konan KS, Kouassi AM, Adingra AA, Gnakri D. 2009. Spatial and temporal variation of fecal contamination indicators in Graand-Lahou lagoon, Côte d'Ivoire. Journal of Applied Biosciences, 23: 1422-1435.

Kouadio N, Dadié A, Adingra A, Aké Y, Djè K. 2011. Biotypes de Escherichia coli isolés des poissons et de l'eau de la lagune de Fresco, Côte d'Ivoire. Journal of Applied Biosciences, 38: 2523-2530.

Kouassi AM, Tidou AS, Kamenan A. 2005. Caractéristiques hydrochimiques et microbiologiques des eaux de la lagune Ebrié, Côte d'Ivoire. Agron. Afr., 17(2): 73-162.

Lefebvre A, Cordier R. 2010. Suivi régional des nutriments (S.R.N.) sur le littoral du Nord, du Pas-De-Calais et de la Picardie. Bilan de l'année 2009. Rapport Ifremer/RST.LER.BL/10.03, p 201.

Mama D, Deluchat V, Bowen J, Chouti W, Yao B, Gnon B, Baudu M. 2011. Caractérisation d'un Système Lagunaire en Zone Tropicale: Cas du lac Nokoué (Bénin). European Journal of Scientific Research, 56(4): 516-528.
Millet M. 1986. Hydrologie et Hydrochimie d'un Milieu Lagunaire Tropical: le Lac Togo. Editions de l'ORSTOM, Collections Etudes et Thèses: Paris; 228.

Mohamed EM, Mohamed F, Serghini A, Blidi SE, Abidi AE, Bennaakam R, Yahyaoui A, Jbilou M. 2008. Impact de l'aménagement hydraulique sur la qualité des eaux et des sédiments de l'estuaire du Loukkos (côte atlantique, Maroc). Bulletin de l'Institut Scientifique, Rabat, Section Sciences de la Terre, 30: 39-47.

Tiekoura KB, Guessennd KAN, Blessa JC, Oussou KR, Ekaza E, Adingra AA, Ouattara GD, Gbonon VC, Houenou P, Dosso M. 2010. Caractérisation Moléculaire des Souches de Vibrio Cholerae Non O1, Non O139 Isolées des Eaux Lagunaires de Grand-Lahou, Côte d'Ivoire. European Journal of Scientific Research, 45(3): 333-345.

Villanueva MC. 2004. Biodiversité et relations trophiques dans quelques milieux estuariens et lagunaires de l'Afrique de l'Ouest : adaptations aux pressions environnementales. Thèse de Doctorat de l'Institut National Polytechnique de Toulouse, Toulouse, $\mathrm{p}$. 246.

Wognin AV, Monde S, Affian K, Coulibaly A, Aka K. 2007. Modèle de circulation des eaux dans l'estuaire du fleuve Bandama en Côte d'Ivoire. Sud Sciences \& Technologies, 15: 5-12.

Wognin V, Monde S, Coulibaly A, Kouassi KL, Adopo L, Affian K, Aka K. 2008. Waters Model Circulation in the Estuary of Bandama. Rivers Flows and Tide condition's Incidence. European Journal of Scientific Research, 19(2): 304-314.

Yao KS, Abé J, Bamba SB, Konan KE, Aka K. 2010. Dynamique d'un périmètre littoral portuaire: la côte de San-Pédro, Sud-Ouest de la Côte d'Ivoire. Revue Paralia, 3: 2.1-2.12. 